\title{
A predictive capability for the aerodynamic deformation of convertible car roofs
}

\author{
J J Knight ${ }^{1 *}$, A D Lucey ${ }^{2}$, and C T Shaw ${ }^{3}$ \\ ${ }^{1}$ School of Engineering and Technology, University of Hertfordshire, Hertfordshire, UK \\ ${ }^{2}$ Fluid Dynamics Research Group, Curtin University, Perth, Australia \\ ${ }^{3}$ Topajka Shaw Consulting Limited, Te Anau, New Zealand
}

The manuscript was received on 10 October 2009 and was accepted after revision for publication on 19 April 2010.

DOI: 10.1243/09544070JAUTO1408

\begin{abstract}
The flow-induced deformation of a membrane in a flow is studied using experimental and computational approaches in a configuration that represents the effect of the aerodynamic load on a convertible car roof. The computational method couples a commercial computational fluid dynamics code with an in-house structural code to predict membrane deformation. A converged statically deformed state, within 1 per cent difference in the displacement variable, is reached after three iterations between the fluid and structural codes. The predictions of membrane deformation are shown to agree well with the experimental results. The key outcome is the demonstration of methodology that will be useful in the engineering design of convertible car roofs.
\end{abstract}

Keywords: convertible roof, fluid-structure interaction, aero-elasticity, membrane, pressure gradient, flexible surface

\section{INTRODUCTION}

The purpose of this note is to communicate a computational methodology that can provide engineering predictions of the deformation or 'bulging' of the material of a convertible car roof due to the aerodynamic loading created by the forward speed of the vehicle. The work presented herein builds upon the fundamental concepts and modelling techniques developed in a previous two-dimensional study by the present authors [1] of the nature of carroof fluid-structure interaction (FSI). Accordingly, this note describes the application and extension of reference $[\mathbf{1}]$ to the three-dimensional FSI system, thereby yielding a modelling tool that can be useful for the engineering design of convertible car roofs.

To establish the methods, the model problem depicted in Fig. 1 is used for experimental investigation and computational modelling. The rigid roof of a quarter-scale $45^{\circ}$ notchback MIRA reference

*Corresponding author: School of Engineering and Technology, University of Hertfordshire, College Lane, Hatfield, Hertfordshire AL10 9AB, UK.

email: j.knight@herts.ac.uk vehicle [2] is replaced by a fabric membrane held along all its edges. The shape that the membrane adopts is determined by the combination of fluidpressure loading with the geometric and material properties of the membrane. The flow field is determined by the geometry of the car to which deformation of the membrane contributes. Thus, fluid and structural mechanics must be coupled to yield a system solution that holds concurrently in both fluid and solid media. The problem studied herein is dominated by the non-linear deformation of the membrane and the mean flow is characterized by the strong pressure gradients that arise from the shape of the roof and the deformation of the membrane.

Linear analyses (see, for example, references [3] to [5]) using ideal uniform mean flow (zero pressure gradient) predict that flexible surfaces (typically a thin flexible plate is modelled) lose their stability to divergence which is supplanted by coupled-mode flutter at higher flow speeds. Non-linear two-dimensional analyses [6, 7], which incorporate deformation-induced tension, show that (linear) divergence instability saturates at a finite amplitude when structural damping is present to give a nearly static 


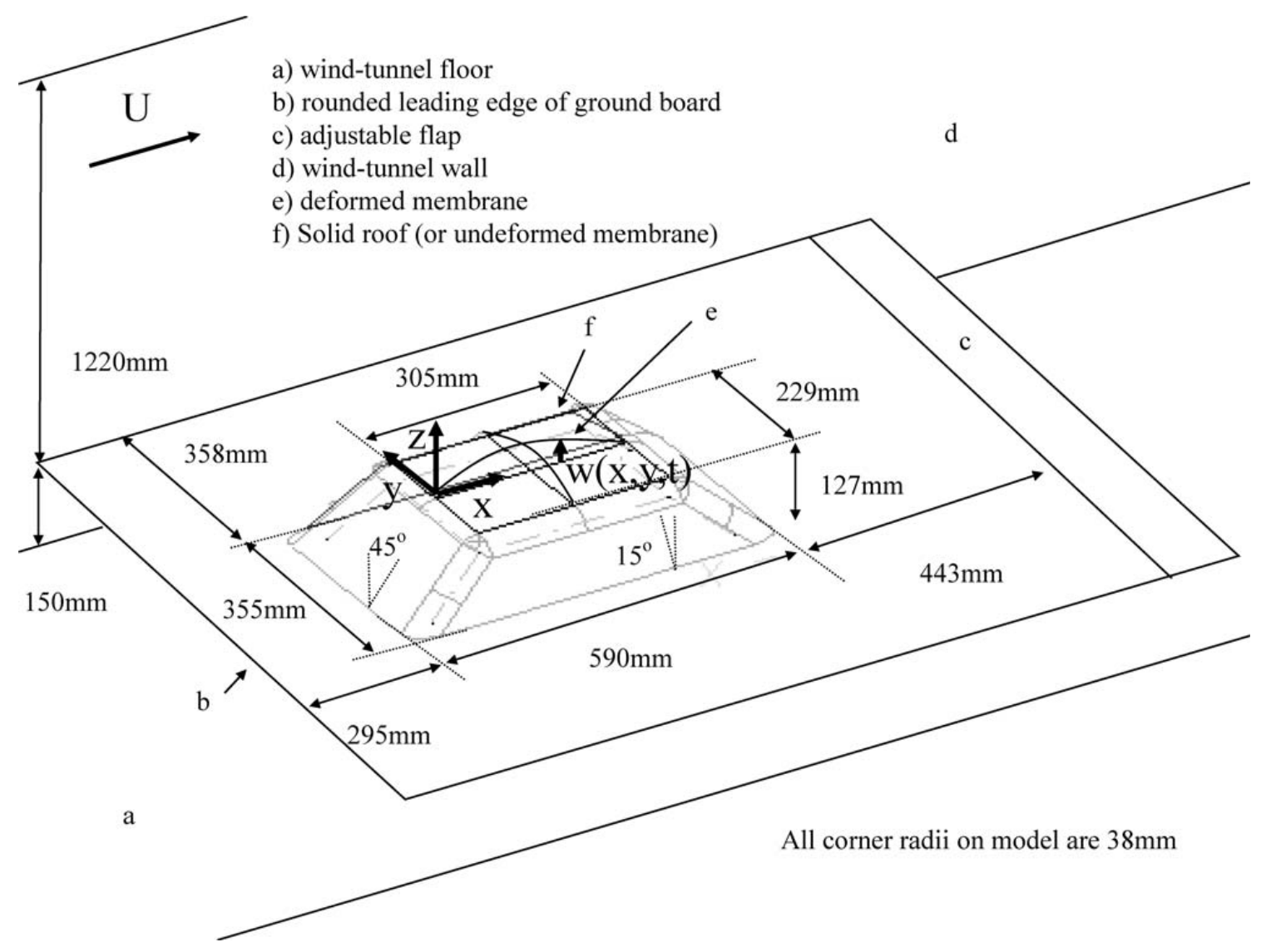

Fig. 1 Schematic diagram of the scale-model car roof used both in the experimental investigation within the wind tunnel and for the computational modelling. Note that gravitational effects acting in the $-z$ direction cause the flexible roof to sag downwards when there is no wind loading

deformation of the flexible surface. Non-linear static divergence in essentially the fundamental mode was also found to characterize the membrane deformation in a previous two-dimensional study of the convertible car-roof FSI system by the present authors $[\mathbf{1}]$. Thus, in the present development, the focus is on the determination of static-equilibrium states in the FSI system. The structural modelling employed is similar to that used in sail design [8] wherein deformations of the membrane are dominated by the fundamental mode. Likewise, the application also requires that flow separation is modelled. For the present car-roof model, separation occurs upstream and downstream of the flexible membrane. The upstream separation occurs owing to rapid changes in geometry typically found at the bonnet-windscreen junction of cars. However, the flow usually reattaches on the windscreen and its effect is to reduce the minimum pressure near the front of the roof line. Downstream separation typically occurs on the rear windscreen of cars; its effect is to modify the upstream mean flow over the membrane, leading to a much reduced suction over the rear of the car roof.

In the statically stable equilibria that are sought, the pressure difference between the external and internal surfaces of the membrane balances the structural restorative forces due to induced tension and any pre-tension. For two-dimensional deformations, the product of tension and membrane curvature balances the local pressure coefficient $[\mathbf{1}, \mathbf{9}]$. In the present three-dimensional work, the membrane is doubly curved and thus it is the combination of curvatures in orthogonal directions with their associated tensions that locally balances the pressure force. This is the basis for the computational modelling developed and experimentally validated in this note.

Below, first the results of an experimental investigation of the car-roof membrane deformation due to its aerodynamic loading are presented. Then these findings are used to validate and assess the accuracy of the computational model that follows. Finally, some recommendations are made on the use of the 
predictive capability as a design tool in automotive engineering.

\section{EXPERIMENTAL INVESTIGATION}

The dimensioned model set-up for wind-tunnel testing is shown schematically in Fig. 1. Since the primary concern is the flow over the roof of a vehicle, only the top half of a MIRA reference vehicle is used. The model was mounted on a ground board spanning the tunnel width to eliminate 'end effects' [10] that can otherwise induce flow around the sides of the ground board. The ground board was located at a height of $150 \mathrm{~mm}$ from the floor of the wind tunnel, well clear of the boundary layer that was measured in the empty wind tunnel to have a displacement thickness of $70 \mathrm{~mm}$. The leading edge of the ground board was rounded to promote attached flow along both of its surfaces. The front of the model was situated a half-model length downstream of the leading edge of the ground board. The boundary layer on the ground board upstream of the model was estimated to have a thickness of $3 \mathrm{~mm}$ using the Blasius solution. Downstream of the model was a three-quarters model length of the ground board, leading to an adjustable flap at the trailing edge of the board. The flap angle was adjusted to ensure an oncoming flow parallel to the ground board upstream of its leading edge using a multi-directional probe. The datum and coordinate system used throughout this note is also shown in Fig. 1. All the experiments were conducted in the University of Warwick's wind tunnel which has a $1071 \mathrm{~mm} \times 1370 \mathrm{~mm}$ working cross-section and turbulence level below 1 per cent. The blockage ratio of the model in the wind tunnel is 3 per cent. Using a wind speed of $12 \mathrm{~m} / \mathrm{s}$ and the model length, the Reynolds number is 480000 .

The fabric membrane of roof was attached by taping it in place. This obviated damage and distortion due to clamping or pin-type fixtures. The tape also sealed the cavity from the external pressure field. Attempts to pre-tension the fabric evenly were unsuccessful and thus the membrane deforms to an inverted dome shape under its own weight. The amount of slackness or 'sag' at the midpoint was recorded as an experimental variable and for use as an initial condition in the computational modelling. The membrane consisted of a poly(vinyl chloride)coated polyester fabric typical of that used for convertible car roofs. It is made up of two series of fibres aligned perpendicular to each other in the warp and weft directions [11]. The former are nearly straight while the latter fibres are woven across these. This matrix is bonded with a resin that prevents rotation of the fibres relative to each other. Weaving requires a longer length of fibres in the weft over the warp direction for a given distance and thus the weft direction yields more readily under tensile loading. Uniaxial testing [12] gave material properties as $E h=78600 \mathrm{~N} / \mathrm{m}$ and $50000 \mathrm{~N} / \mathrm{m}$ in the warp and the weft directions respectively, where $E$ is the elastic modulus and $h=0.29 \mathrm{~mm}$ was the thickness of the membrane. Throughout the present investigation the material was oriented with the warp aligned with the streamwise $x$ direction. The model dimensions and material choice for a typical wind-tunnel speed $U=12 \mathrm{~m} / \mathrm{s}$ yield a Cauchy (or Hooke) number of $6.85 \times 10^{-4}$, using the definition [1] $C a=\rho U^{2} L /$ $(E h)$, where $L$ is the streamwise length of the flexible roof. This correlates well with real convertible-car FSI applications to give close dynamic similarity.

The measured variations in the membrane midpoint deflection with wind speed are presented in Fig. 2 for different values of the initial membrane slackness. The data were obtained using a photogrammetric technique with a single digital camera. The camera was aligned with the spanwise centreline of the model and at a height above the roof level to give a $5^{\circ}$ elevation angle. Additional images were captured at zero elevation to check any dependence of the results upon the chosen elevation angle. Images were imported into the AutoCAD software package to extract quantitative deflection data using the known streamwise dimension of the roof as the reference scale. The detailed error analysis of the experimental technique presented in reference [13] predicts an absolute maximum error within the range from $+0.4 \mathrm{~mm}$ to $-0.5 \mathrm{~mm}$; this translates to an accuracy of between +8 per cent and -10 per cent for a typical deformation of $5 \mathrm{~mm}$. The wind-loading cycle was repeated three times to assess repeatability; deflections were found to be within 4 per cent of each other and the initial slackness measurements within 7 per cent of each other. These variations are represented by the error bars in Fig. 2. For the range of flow speeds presented in Fig. 2, the membrane was observed to settle into a static equilibrium with its shape of deformation dominated by the fundamental mode of the membrane. As would be expected, the amplitudes of the deformation increase with increasing wind speed. The contribution of the initial slackness to the amplitude of deformation is seen to decrease as the wind speed is increased. This is because the aerodynamic load increasingly becomes the cause of 


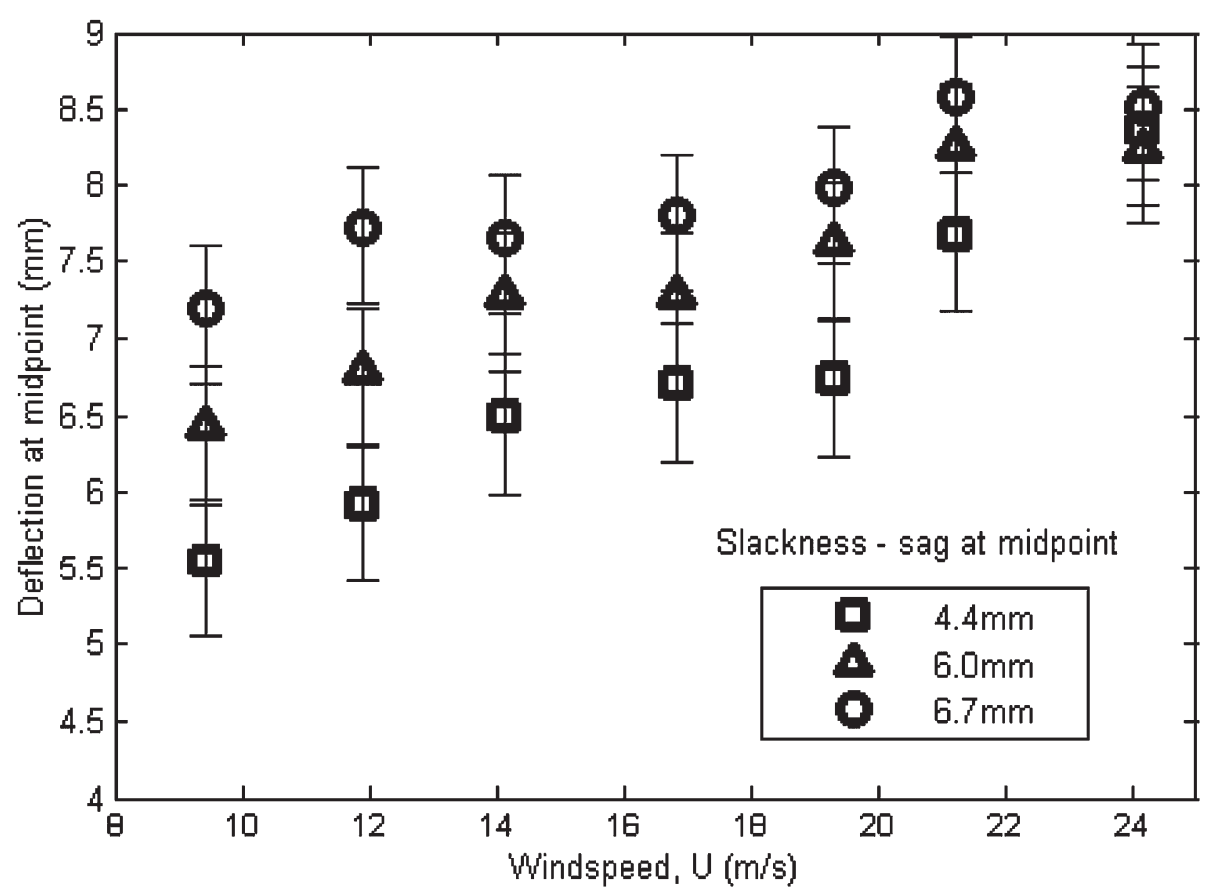

Fig. 2 Variation in the membrane midpoint deformation with the wind speed (the forward speed of the vehicle) for different conditions of the unloaded membrane

membrane deformation. The convergence of the data sets in Fig. 2 also indicates that membrane deformation makes only a minor adjustment to the flow-field curvature created by the overall shape of the roof. This is further borne out by the computational results presented in section 3.4 below.

\section{COMPUTATIONAL INVESTIGATION}

A computational model is now devised and evaluated to predict the membrane deformations measured experimentally in order to develop an analysis tool useful for the design of convertible car roofs. Given that the aerodynamically loaded membrane settles into a static equilibrium, the time dependence is neglected; this approach is typical of sail design (see, for example, reference [8]). Thus, the balance between the structural and fluid forces acting on the membrane is enforced according to

$$
-T_{\mathrm{I} x}(w) \frac{\partial^{2} w}{\partial x^{2}}-T_{\mathrm{I} y}(w) \frac{\partial^{2} w}{\partial y^{2}}=\Delta P
$$

expressed in terms of the vertical displacement field $w(x, y)$ of the deformation and where $T_{\mathrm{I} x}(w)$ and $T_{\mathrm{I} y}(w)$ are the aggregated warp- and weft-fibre tensions respectively induced by the pressure loading $\Delta P$. This simple FSI model omits the smaller contributions of flexural rigidity to the structural forces. The omission of a tension cross-term is justified by the near independence between deformations of the perpendicular fibres that constitute the material. Also neglected are the effects of geometric non-linearity because the amplitude-towavelength ratio of the deformations is small, and the effects of fluid friction and in-plane deformation because these are typically an order of magnitude smaller than the out-of-plane effects modelled herein (see, for example, reference [14]).

\subsection{Flow-field computation and evaluation}

The uses of potential-flow (with and without a wake shear layer) and Navier-Stokes solvers have been considered in the development of the methodology and assessed for the corresponding two-dimensional problem presented in reference [1]. The pressure distributions were in good agreement over the flexible roof apart from in the region near its downstream edge; this was due to the upstream influence of the flow separation (absent or only approximately modelled by the potential-flow solver) that occurs further downstream over the rear screen. Accordingly, in the present work a NavierStokes solver is used to compute the flow field. Specifically, the commercial computational fluid dynamics (CFD) code StarCD with MARS discretization [15] and the SIMPLE algorithm [16] are used. 
Both the standard $k-\varepsilon$ turbulence model [17] and renormalization group (RNG) variant $[\mathbf{1 8}]$ were implemented and assessed. The RNG $k-\varepsilon$ model has an extra term in the dissipation equation to account for distortion of the mean flow; Baxendale $e t$ al. [19] reported improved flow predictions in separated or recirculating flow. Large eddy simulation can capture separation more accurately but at significantly greater computational cost and therefore it has not been used. The engineering design goal of the present work and the need to compute repeatedly the flow field in the FSI iteration account for the choice of workability with sufficient accuracy.

Mesh generation employed the Rhinoceros NURBS modelling software [20] to create surfaces from which unstructured tetrahedral meshes were extruded using ICEM TETRA [21]. This approach is also workable in real-car applications that may feature more complex geometries. The base mesh used herein contained 256881 cells, biased towards the model surface and regions of high curvature so as to resolve boundary layers and high pressure gradients. Only half of the domain is modelled as a symmetry plane is applied about the longitudinal centre-line of the model. The plane of the ground board in the experiments is used as the lower boundary, and the wind-tunnel wall and roof are used for the side and upper boundaries respectively. The inlet is located one model length ahead of the model, and the outlet three model lengths behind the model. Standard no-slip and no-flux wall boundary conditions are specified at the tunnel walls, ground board, and model surface.

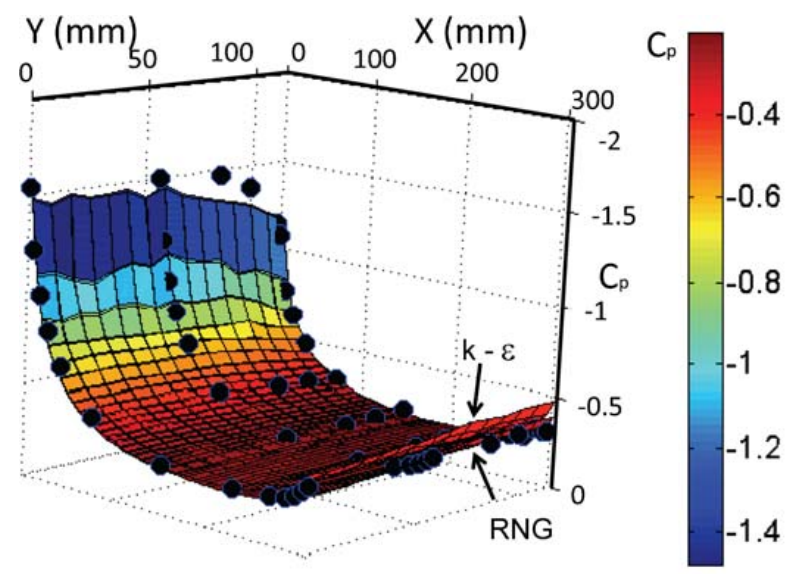

(a)
The solid curve in Fig. 3(a) show the computed pressure field $\left(C_{p}=\left[p(x, y)-p_{0}\right] / \frac{1}{2} \rho U^{2}\right.$, where $p_{0}$ is the upstream static pressure) acting on the top of the model for a rigid roof at a wind speed $U=12 \mathrm{~m} / \mathrm{s}$. Note that only one streamwise half is shown, owing to the symmetry plane, and the origin of the axes is defined in Fig. 1. To validate the computation, the pressure field was measured experimentally by replacing the membrane with a rigid insert in which were a number of pressure tappings; the measurements are presented as full circles in Fig. 3(a). The same computed and experimental pressure data along the longitudinal centre-line $(y=0)$ are shown in Fig. 3(b).

A negative pressure (or suction) is seen to act over the whole roof. The spanwise pressure variation is small but visible both at the front of the roof because of flow around the side of the model and to a lesser extent near the rear of the roof. The latter is caused by the flow which departs from the sides of the model, creating a weak longitudinal vortex in the wake [22]. The longitudinal pressure gradient is greatest near the leading edge of the flat-roof section as the pressure recovers from the favourable pressure gradient created by the windscreen. A decrease in the pressure occurs near the rear of the roof owing to flow acceleration around the rear corner of the roof, after which the flow separates from the rear screen. The computations presented in Fig. 3 show that, while both the standard and the RNG $k-\varepsilon$ turbulence models give good agreement with the experimental results for the upstream region of roof, the standard model is inferior near its rear. Accord-

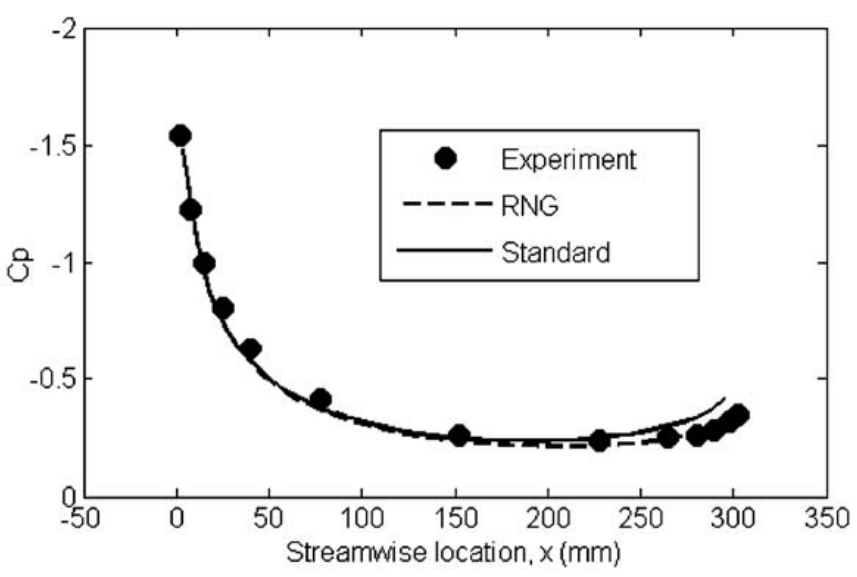

(b)

Fig. 3 Computed pressure field over the top of the rigid (undeformed) roof of the car including discrete data experimental measurements (•): (a) orthographic view of the car roof; (b) along the longitudinal centre-line. Note that the results for the standard model and the RNG $k-\varepsilon$ turbulence model are indistinguishable except where labelled near the downstream end of the roof 
ingly, the RNG $k-\varepsilon$ turbulence model was adopted for the FSI modelling.

\subsection{Structural model}

For the structural side of equation (1), the onedimensional line-element model of reference [1] is extended to model a membrane surface consisting of a network of orthogonal, but non-interactive, elements in the warp and weft directions. Each line element is assumed to have constant tension acting along its length and the resulting surface mesh is additionally used to support a finite difference scheme for the spatial derivatives in equation (1). For a given load, a fixed-point iteration scheme, based upon a dynamic extension of equation (1), is used to proceed in both space and time until the motion decays and deformation shape settles into the solution of equation (1); details of the basic algorithm have been presented in reference [1]. To reduce computational costs, the maximum deflection is assumed to lie on the longitudinal centre-line and a symmetry condition is applied that is consistent with the CFD approach. Thus, the condition of zero transverse gradient of the membrane surface is enforced along the longitudinal centre-line in addition to the hinged-attachment conditions along its fixed edges and pressure correction conditions as used in reference [1]. The incorporation of initial slackness proceeds as a straightforward extension of the method described in reference [1]; the initial sagged shape is generated by applying a uniform pressure load to mimic that of the membrane's self-weight. For a fixed pressure loading, convergence of the structural solver is considered to be achieved when the difference of deflection between successive time iterations falls to within 0.1 per cent of the displacement variable.

\subsection{Coupled fluid-structure interaction model}

The complete FSI model is assembled from the CFD and structural solvers described above. The external pressure field for an undeformed flexible membrane is computed. The pressure difference $\Delta P(x, y)$ across the membrane is evaluated on the basis of a known interior pressure (measured in the experimental investigation) and used in equation (1). The structural solver is then used to determine the static deformation associated with that pressure field. The flow domain is then remeshed to account for the membrane deformation and the pressure field is corrected. Thus, an iterative procedure between the fluid and the structure is established that continues until both the computed pressure field and the membrane deformation converge to that of the static equilibrium sought. The convergence criterion applied was that less than a 1 per cent change in the membrane variable $w(x, y)$ occurs for subsequent iterations. This partitioned approach to FSI modelling is appropriate for the present problem because the goal is to predict a final static equilibrium as opposed to continuous dynamic interactions between fluid and structure.

\subsection{Computation and evaluation of roof deformation due to wind loading}

Figure 4 presents the computed deformation of the roof membrane at a wind speed of $12 \mathrm{~m} / \mathrm{s}$; the surface plot in Fig. 4(a) principally illustrates its streamwise profile while the solid curves in Fig. 4(b) show its spanwise profile at three streamwise locations, namely $x=0.25 L, 0.5 L$, and $0.75 L$, where $L$ is the streamwise length of the membrane. Convergence to this final deformation was achieved after three iterations between the fluid and structural solvers. The initial (unloaded) deformation of the membrane was input as having a $4.4 \mathrm{~mm}$ sag at its centre. Note that only half of the membrane is presented because symmetry about the membrane centre-line is assumed in the modelling.

Figure 4(a) also includes the midline deflections measured in the experimental investigation that generated the data point at $12 \mathrm{~m} / \mathrm{s}$ for $4.4 \mathrm{~mm}$ of sag in Fig. 2. Good agreement is found between the magnitudes of the computed prediction and the measured deformation. There is agreement in the overall shape of the predicted and measured deformations but the latter has its maximum closer to midchord. This difference may be due to the omission of the fluid shear load on the structure in the present computational model. As expected, upon the consideration of the rigid-roof pressure field presented in Fig. 3, the peak of midline deformation is biased towards the leading edge of the membrane owing to the high values of suction in that region. Computed pressure fields (not presented here) that include the membrane deformation show the midline pressure distribution to be very similar to that seen in Fig. 3, but with an approximately 10 per cent increase in the magnitude of $C_{p}$ over the region $x=50-280 \mathrm{~mm}$. Thus, while the membrane deformation does make a contribution (a calculated increase of 9.8 per cent in the total pressure force acting on the membrane), the principal source of the pressure field that deforms the 


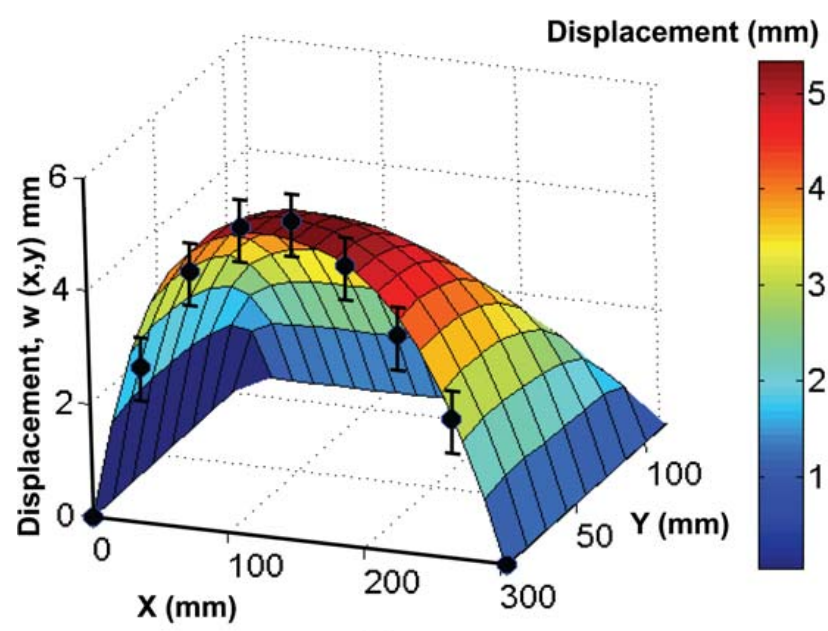

(a)

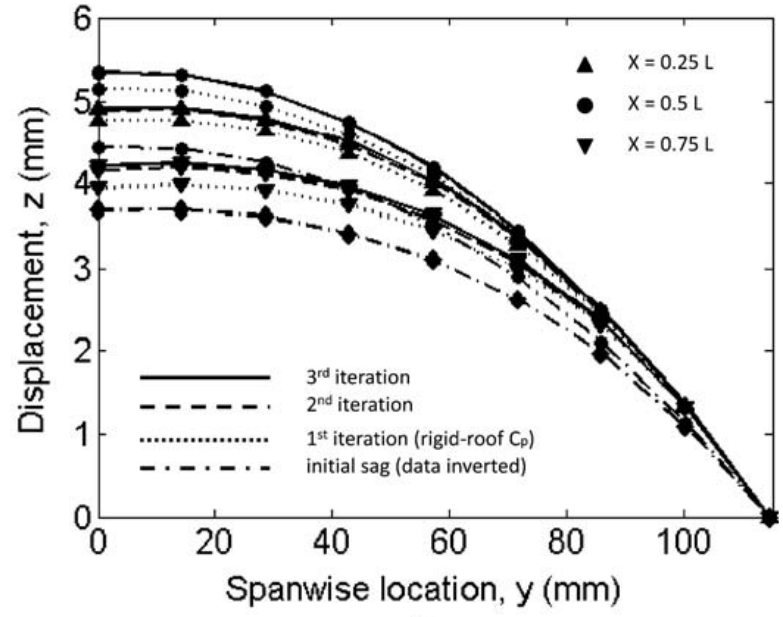

(b)

Fig. 4 Aerodynamically loaded deformation of the membrane roof of the car at $U=12 \mathrm{~m} / \mathrm{s}$ : (a) orthographic view of converged computed profile including experimentally measured deformation ( ) along the longitudinal centre-line; (b) computed transverse roof profile at three different streamwise locations, showing the initial sagged condition (data inverted) and intermediate steps in the iterative process. In both (a) and (b), a stretched vertical scale is used to highlight the shape of the deformed membrane

membrane is the overall shape of the car roof. Figure 4(b) further explores this theme. In addition to the final deformation of the membrane profile, intermediate results during the iterative process are presented. The initial condition (no wind loading) of the sagged membrane is plotted with its data inverted so that the deformation due to the wind loading can be assessed; thus, for example, while the membrane midpoint has a final deformation of $5.4 \mathrm{~mm}, 4.4 \mathrm{~mm}$ of this is due to the original slackness in the membrane. The data in this figure also show the convergence rate of the FSI solution process; the deformations at the second and third iterations are almost indistinguishable. Finally, it can be seen that the first iteration, which employed the rigid-roof pressure field, yields a flow-induced deformation that is approximately 70 per cent of the final converged result. This then suggests that a reasonable engineering approximation of the deformation of a convertible car roof could be based upon the fact that the pressure field acts on the basic design shape of the vehicle.

\section{CONCLUSIONS}

An experimental investigation has been conducted to determine the deformation due to the wind loading of a model convertible car roof. For the range of flow speeds studied, the roof always settles into a statically deformed shape determined by the wind loading. The experimental findings have been used to develop and validate a computational model of the specific fluid-structure interaction. Within this model, the pressure loading is determined by solving the Navier-Stokes equations with an RNG $k-\varepsilon$ turbulence model, while the structural deformation of a fabric membrane that represents the convertible part of the roof is found using a line-element model. The coupled FSI algorithm is robust, the flexible roof converging to a statically deformed state that agrees well with the experimental results in typically three iterations between fluid and structural solvers. However, it is also shown that an engineering approximation, within 30 per cent of the actual results, can be achieved with just one iteration using the pressure field acting on the basic car shape. The dynamic similarity of the FSI between the experimental model adopted and that of a full-scale convertible car suggests that the computational model validated in this technical note would serve as a practicable design tool in industry.

(c) Authors 2010

\section{REFERENCES}

1 Knight, J. J., Lucey, A. D., and Shaw, C. T. Fluidstructure interaction of a two-dimensional membrane in a flow with a pressure gradient with application to convertible car roofs. J. Wind Engng Ind. Aerodynamics, 2010, 98(2), 5-72.

2 Carr, G. Validation of CFD codes for predicting aerodynamic performance. Automot. Engr, October-November 1992, 46-49. 
3 Weaver, D. S. and Unny, T. S. The hydroelastic instability of a flat plate. Trans. ASME E, J. Appl. Mech., 1971, 37, 823-827.

4 Ellen, C. H. The stability of simply supported rectangular surfaces in uniform subsonic flow. Trans. ASME E, J. Appl. Mech., 1973, 95, 68-72.

5 Lucey, A. D. and Carpenter, P. W. The hydroelastic stability of three-dimensional disturbances of a finite compliant panel. J. Sound Vibr., 1993, 165, 527-552.

6 Lucey, A. D., Cafolla, G. J., Carpenter, P. W., and Yang, M. The non-linear hydroelastic behaviour of flexible walls. J. Fluids Structs, 1997, 11, 717-744.

7 Peake, N. Nonlinear stability of a fluid-loaded elastic plate with mean flow. J. Fluid Mech., 2001, 434, 101-118.

8 Cyr, S. and Newman, B. Flow past two-dimensional membrane aerofoils with rear separation. $J$. Wind Engng Ind. Aerodynamics, 1996, 63, 1-16.

9 Newman, B. G. and Goland, D. Two-dimensional inflated buildings in a cross wind. J. Fluid Mech., 1982, 117, 507-530.

10 Rae Jr, W. H. and Pope, A. Low-speed wind tunnel testing, 2nd edition, 1984 (John Wiley, New York).

11 Hepple, P. More stretch, more speed. Seahorse, May-June 1984, 31-33.

12 Choong, L.-W. Numerical formulation and modelling of fabric convertible hoods under aerodynamic loading. MPhil Thesis, University of Warwick, Coventry, UK, 2002.

13 Knight, J. J. Development of a flow-structure interaction methodology applicable to a convertible car roof. PhD thesis, University of Warwick, Coventry, UK, 2003.
14 Tetlow, G. A. and Lucey, A. D. Motions of a cantilevered flexible plate in viscous channel flow driven by a constant pressure drop. Commun. Numer. Meth. Engng, 2009, 25, 463-482.

15 Star-CD v3.05 user guide and methodology manual, 1997 (Computational Dynamics Ltd, London).

16 Patankar, S. V. and Spalding, D. B. A calculation procedure for heat, mass and momentum transfer in three-dimensional parabolic flows. Int. J. Heat Mass Transfer, 1972, 15, 1787-1806.

17 Jones, W. P. and Launder, B. E. The prediction of relaminarization with a two-equation model of turbulence. Int. J. Heat Mass Transfer, 1972, 15, 301-314.

18 Yakhot, V., Orszag, S. A., Thangam, S., Gatski, T. B., and Speziale, C. G. Development of turbulence models for shear flows by a double expansion technique. Phys. Fluids A, 1992, 4(7), 1510-1520.

19 Baxendale, A. J., Graysmith, J. L., Howell, J. P., and Haynes, T. Comparisons between CFD and experimental results for the Ahmed reference model. In Proceedings of the Royal Aeronautical Society Conference on Vehicle Aerodynamics, London, UK, 18-19 July 1994, pp. 30.1-30.11.

20 Rhinoceros NURBS modelling for windows, user guide V1.0, 2000 (Robert McNeel and Associates, Seattle, Washington).

21 ICEM CFD/CAE 3.3.3 user manuals, online edition, 1998 (ICEM CFD Engineering, Canonsburg, Pennsylvania).

22 Hucho, W.-H. Aerodynamics of road vehicles, 4th edition, 1998 (SAE International. Warrendale, Pennsylvania). 